\title{
Efecto inmunosupresor de la infección por Trypanosoma musculi (Mastigophora: Trypanosomatidae) en la toxoplasmosis experimental
}

\author{
Loretta Piccolo-Johanning, Vivian Kellerman-Guterman, Idalia Valerio-Campos \& Misael \\ Chinchilla-Carmona \\ Departamento de Investigación y Cátedra de Parasitología Médica, Universidad de Ciencias Médicas (UCIMED), \\ San José Costa Rica, América Central; loretta_piccolo@hotmail.com, vivik01@hotmail.com, valerioci@ucimed.com, \\ chinchillacm@ucimed.com
}

Recibido 12-IV-2012. Corregido 10-XII-2012. Aceptado 24-I-2013.

\begin{abstract}
Immunosuppressor effect of Trypanosoma musculi (Mastigophora: Trypanosomatidae) on experimental toxoplasmosis. The immunosuppression caused by species of the gender Trypanosoma has been widely documented. The influence over experimental infections with Toxoplasma gondii is evident when using Trypanosoma lewisi, a natural parasite of white rats. We decided to test the effect of Trypanosoma musculi from mice, an organism with very similar biological characteristics to T. lewisi, to see if this trypanosomatid could induce a similar effect. Four groups of Swiss mice were inoculated with T. musculi previously to infection with T. gondii, and we determined the survival of the animals, as well as the number of cysts developed in the brain of survivors. We isolated and tested different strains of $T$. gondii from different sources. In a first experiment, the animals were previously inoculated with $T$. musculi at different times prior to the infection with Toxoplasma; this allowed us to determine that the immunosuppression process resulted more evident when T. musculi inoculation was made four days before. In a second experiment, we used different inoculi dose and found that it did not influenced the process. Furthermore, the results were negative when evaluating if the amount of the inoculated trypomastigote influenced the process. In order to demonstrate if there were differences in the immnosuppressive effect, related to Toxoplasma strains, groups of mice were inoculated with brain cysts of TFC, TLP, TLW and TBT strains. Excluding the TLP strain, that resulted to be very pathogenic regardless the previous inoculation with $T$. lewisi, the other strains kept the same pattern of immunosuppression in mice, whose survival time was shorter as the presence of cysts in the brain was higher. These observations were in agreement with an experimental immunosuppression model, associated with immunosuppressive diseases, specially cancer and AIDS. Rev. Biol. Trop. 61 (2): 981-990. Epub 2013 June 01.
\end{abstract}

Key words: Toxoplasma gondii, inmunosuppression, Trypanosoma musculi, experimental model, inmmunity.

La prevalencia de infecciones por Toxoplasma gondii en el ser humano es de 5-90\%, dependiendo de la zona geográfica (Bojar \& Szymanska, 2010). En Costa Rica, la seroprevalencia es de un 58\% (Zapata et al. 2005). La presentación aguda de la parasitosis es poco frecuente, por lo que en la mayoría de los casos cursa como una enfermedad crónica, debido a que la acción inmunológica del hospedero rápidamente bloquea la reproducción del parásito, el cual forma quistes en músculo y cerebro especialmente, que usualmente se mantienen latentes a lo largo de la vida del hospedero (Dubey \& Jones 2008). En el caso de individuos inmunodeprimidos ya sea por causas naturales (cáncer, infecciones con VIH, Lupus, entre otros) (Sitoe et al. 2010) o por tratamientos excesivos con inmunosupresores, el parásito puede activarse produciendo una toxoplasmosis aguda (Holland 2003, Elmore et al. 2010, Solene et al. 2010).

Los fenómenos de inmunosupresión mencionados anteriormente se han estudiado experimentalmente, tanto para $T$. gondii (Kankova et al. 2010 \& Da Silva et al. 2010) como para otros parásitos intracelulares (Uzonna et 
al.1998, Da Silva et al. 2010, Fazzani et al. 2011). En el caso de la toxoplasmosis, se han realizado varios estudios que revelan claramente esta situación, especialmente en cuanto a infecciones concomitantes se refiere. El hecho de que la infección con tripanosomas africanos induzca procesos de inmunosupresión claramente demostrados (Darji et al. 1992, Uzonna et al.1998) dio origen a varios estudios en las ratas blancas (Rattus rattus). Estos animales presentan una clara resistencia natural a la toxoplasmosis, la cual siempre se manifiesta en ellos como una enfermedad crónica (Chinchilla et al. 1981,1982, 1985), independientemente de la cepa del parásito y la edad de los animales (Guerrero et al.1995, Albright \& Albright 1991). En tales estudios, se demostró que el Trypanosoma lewisi, un parásito normal de la rata, que no provoca patología sobre el animal (ya que la infección es autolimitada), induce un efecto inmunosupresor importante que activa la toxoplasmosis en estos animales. Este efecto se produce tanto al infectar los animales vía intraperitoneal con taquizoitos, la forma más activa del parásito, como con ooquistes, estado evolutivo de infección oral del T. gondii (Guerrero et al. 1997). T. musculi cuyo hospedero es el ratón (Mus musculus), pertenece al mismo grupo Stercoraria de T. lewisi; además presenta prácticamente las mismas características biológicas e inmunológicas del tripanosomátido de ratas (Viens et al. 1974). Por esta razón consideramos que el mismo efecto inmunosupresor podría presentarse en ratones infectados con T. musculi. Estos últimos animales son susceptibles a la mayoría de las cepas de $T$. gondii por lo que el estudio trata de agudizar la infección crónica o intensificarla en los ratones infectados con una cepa adecuada del parásito. Los estudios experimentales que han tratado de dilucidar estos supuestos, constituyen la base de este estudio.

\section{MATERIALES Y METODOS}

Animales: En todos los experimentos se usaron ratones machos y hembras (Mus musculus cepa Swiss), con un peso promedio de $23 \mathrm{~g}$. Para los experimentos varios grupos de cuatro a cinco animales se mantuvieron bajo las condiciones recomendadas por la ley No. 7451 sobre Bienestar de los Animales (La Gaceta n ${ }^{\circ} 44$ del 04 marzo de 1998 de Costa Rica), con suministro de alimento y agua ad libitum por un máximo de 30 días, tiempo límite de todos los experimentos.

Parásitos: Se emplearon las cepas 30182 de T. musculi de la American Type Culture Collection (ATCC) y las cepas de $T$. gondii siguientes, caracterizadas como cepas de tipo crónico: TFC, TLW, TLP aisladas de las heces de Felis catus, Leopardus wiedii y Leopardus pardalis respectivamente, así como la cepa TBT aislada de una muestra de carne de un bovino (Bos taurus). La cepa de T. musculi se mantuvo en el laboratorio inoculándola vía intraperitoneal (i.p.) semanalmente en ratones Swiss; las de $T$. gondii, por ser de tipo crónico, se traspasaron cada dos a tres meses, por inoculación de quistes del parásito en el tejido cerebral a ratones sanos. Para los procesos de inoculación, sacrificio y disección se siguieron los protocolos establecidos por la ley mencionada.

Modelo experimental: Grupos de cuatro o cinco ratones fueron inoculados vía intraperitoneal con $0.2 \mathrm{~mL}$ de dilución con $10^{6} \mathrm{de}$ tripomastigotos de T. musculi, obtenidos por sangrado de animales previamente infectados, entre los meses de Julio 2010 a Agosto 2011. Otro grupo de ratones, con características similares, y que no fue infectado con el tripanosomátido, sirvió como control. Después de un tiempo establecido para cada experimento, que varió entre cuatro y siete días, los ratones de todos los grupos fueron inoculados i.p con $0.2 \mathrm{~mL}$ de quistes de $T$. gondii. Estos quistes fueron obtenidos al extraer cerebros de ratones previamente infectados con las diferentes cepas; la cantidad a inocular se determinó al establecer el número de quistes por gramo de tejido. Se prepararon diluciones del macerado del cerebro, para establecer el número de quistes a inocular, de acuerdo con los lineamientos 
específicos de cada experimento (Holst \& Chinchilla 1990, Guerrero et al. 1997). Posteriormente, se hizo el control de supervivencia de los animales, con un tiempo máximo y final del experimento de 30 días. Después del mes de infección, los animales supervivientes fueron sacrificados para determinar en ellos el número de quistes por gramo de cerebro, de acuerdo con estudios previos (Holst \& Chinchilla 1990). Todos estos datos y los relativos a la supervivencia, fueron evaluados al comparar los resultados obtenidos en los ratones inoculados y sin inocular con T. musculi.

Con base en los lineamientos anteriores se procedió a realizar los siguientes experimentos específicos de comprobación de la hipótesis planteada.

1. Con el objeto de comprobar si T. musculi induce un efecto inmunosupresor sobre la toxoplasmosis experimental, cuatro grupos de cuatro ratones fueron inoculados con $10^{6}$ tripomastigotos por animal; el quinto grupo no fue inoculado y sirvió como control. Posteriormente, los grupos uno, dos, tres y cuatro fueron infectados con 50 quistes de $T$. gondii (cepa TLW) después de cuatro, cinco, seis y siete días respectivamente, y el grupo cinco recibió los quistes a los cuatro días, todo de acuerdo con la metodología descrita en trabajos previos (Guerrero et al. 1997). En el experimento que se extendió por 30 días, se usaron 20 ratones en total.

2. En el experimento anterior se determinó que existía algún efecto inmunosupresor sobre la toxoplasmosis experimental y que los mejores resultados se obtenían cuando la infección con el tripanosoma se realizaba cuatro días antes de inducir la toxoplasmosis experimental. Para establecer si el número de tripanosomas infectados era un factor importante en el proceso, se procedió a inocular seis grupos de cinco ratones con $4 \times 10^{7}, 4 \times 10^{5}, 4 \times 10^{3}, 4 \times 10^{1}$, $4 \mathrm{y}<4$ tripomastigotos por ratón en cada grupo, respectivamente; el grupo siete no fue inoculado con este parásito. Después de cuatro días, los animales de los siete grupos fueron infectados con aproximadamente 50 quistes de T. gondii (cepa TLW). El total de animales usados fue de 35 en este experimento que se prolongó por 30 días.

3. Se realizó un tercer experimento para establecer si el efecto inmunosupresor presentaba variantes debidas al tipo de cepa de $T$. gondii. Para cada una de las cepas de este parásito, TFC, TLW, TLP y TBT se prepararon dos grupos de cinco ratones, uno de los grupos se infectó previamente con T. musculi y el otro no; después de cuatro días los dos grupos se inocularon con 25 quistes de $T$. gondii de la cepa respectiva. El inóculo fue más bajo con la idea de contar con mayor número de sobrevivientes y establecer así las diferencias del caso en cuanto a la cantidad de quistes en el cerebro de los animales. De acuerdo con el número de grupos establecidos, se inocularon un total de 40 ratones, algunos de los cuales murieron antes de los 30 días, tiempo máximo de duración del experimento.

Para todos los tres experimentos específicos anteriormente descritos, una vez realizadas las inoculaciones se procedió de acuerdo con el modelo experimental general en el control de supervivencia y determinación del número de quistes por gramo de cerebro de los supervivientes.

Los datos correspondientes a la determinación de las diferencias entre promedios del número de quistes de $T$. gondii en animales con o sin previa infección con $T$. musculi, fueron analizados con estadística paramétrica y no paramétrica (prueba de suma de rangos Wilcoxon-Mann-Whitne para ambos casos). Para analizar la supervivencia de los animales en los diferentes experimentos se utilizó la prueba de estimador de Kaplan-Meier y la prueba t student, ampliamente conocida y más adecuada para experimentos con un número pequeño de datos. 


\section{RESULTADOS}

En el primer experimento se demostró que la inoculación previa con $T$. musculi reduce la supervivencia de los animales infectados con T. gondii (Fig. 1). En efecto gran cantidad de taquizoítos fueron encontrados en el exudado peritoneal de los ratones que murieron antes de los 30 días. El menor promedio de supervivencia se presentó en aquellos ratones en que la infección con el tripanosoma fue de cuatro días antes de la inoculación con $T$. gondii $(\mathrm{p}<0.05)$,

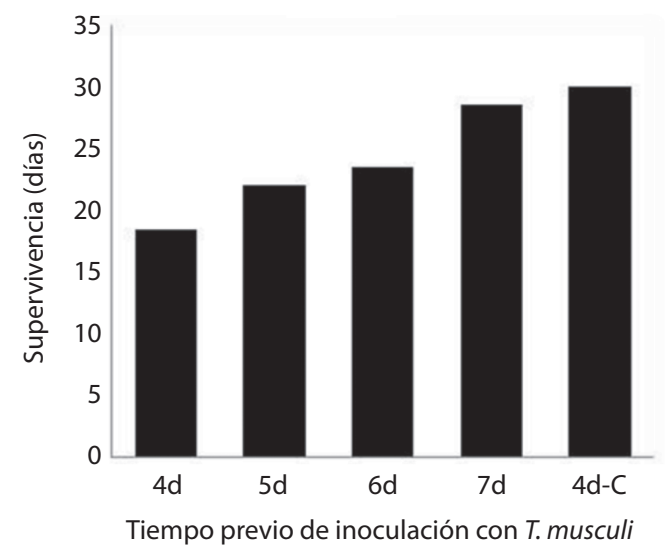

Fig. 1. Supervivencia de ratones infectados con T. gondii previa inoculación con $T$. musculi.

Fig. 1. Survival time of mice infected with $T$. gondii after inoculation with T. musculi. aumentando proporcionalmente con el número de días de infección previa $(\mathrm{p}<0.05)$. Por lo tanto, se estableció el tiempo previo de cuatro días para la infección con T. musculi para los siguientes experimentos.

Aunque fueron pocos los datos obtenidos, porque varios animales no sobrevivieron los 30 días establecidos como final del experimento, el número de quistes/g de cerebro en los animales no inoculados con T. musculi, 16561, fue menor que aquellos que sobrevivieron de los grupos con tres y cuatro días de inoculación previa con el tripanosoma, 38720 y 21069 , respectivamente.

Al estudiar la influencia de la cantidad de tripanosoma inoculados con el efecto inmunosupresor observado, se determinó que todos los animales infectados solamente con $T$. gondii sobrevivieron los 30 días, mientras que los otros presentaron promedios de supervivencia que oscilaron entre los 20 y 26 días (Fig. 2). Por lo tanto, el efecto inmunosupresor se mantuvo ( $\mathrm{p}$ entre $<0.025$ y 0.05 ), pero no existieron diferencias significativas aplicables a la cantidad de tripomastigotos inoculados ( $\mathrm{p}>0.05)$. El número de quistes por gramo de cerebro en los ratones control sobrevivientes (datos no presentados) fue mucho menor (3034), que el de los animales inmunosuprimidos (alrededor de 11000$)$.

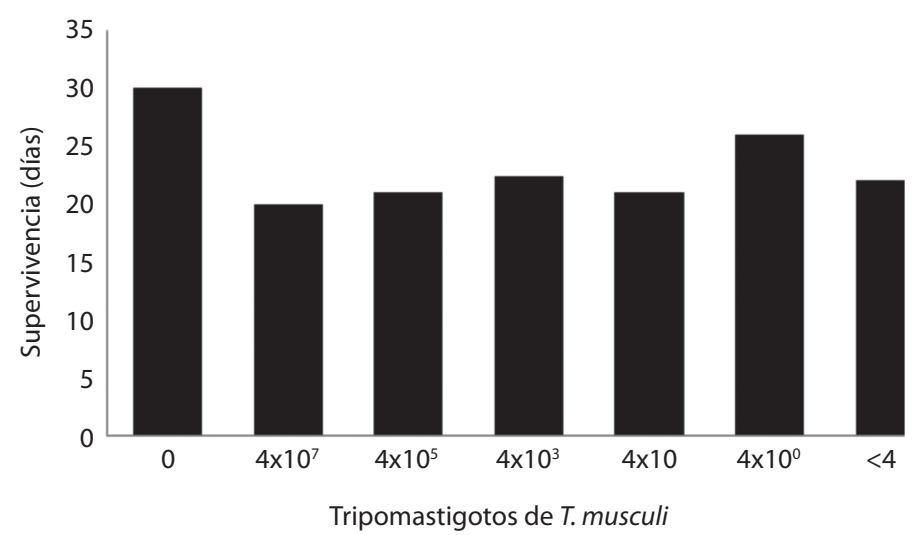

Fig. 2. Supervivencia de ratones infectados con T. gondii previa inoculación con cantidades diferentes de tripomastigotos de T. musculi.

Fig. 2. Survival time of mice infected with $T$. gondii previously inoculated with different number of $T$. musculi trypomastigotes. 
En el estudio con diferentes cepas de $T$. gondii, la supervivencia en general fue más elevada, tal y como se esperaba, al rebajar el número de quistes inoculados. La excepción a esta regla fue la cepa TLP, cuya actividad patológica ha sido evidente a través de otras observaciones (datos no publicados). En efecto, todos los animales inoculados murieron antes de 30 días, independientemente de la infección previa o no con el tripanosoma (Fig. 3). Por los datos obtenidos en cuanto a supervivencia no se deduce ninguna diferencia, pero cuando se hizo el análisis de quistes por gramo de cerebro en los supervivientes, se notó que para las tres cepas, los animales del grupo control (no inoculados con T. musculi), siempre presentaron un menor número de quistes (Fig. 4) y las diferencias estadísticas $(\mathrm{p}<0.011)$ fueron significativas.

\section{DISCUSION}

Los procesos de inmunosupresión son importantes en el sentido que significan un debilitamiento en las defensas naturales de los seres vivientes, lo que los convierte en terreno fértil para el desarrollo de enfermedades causadas fundamentalmente por virus, bacterias y hongos (Manzano-Alonso \& CastellanoTortajada 2011, Duncan \& Wilkes 2005), pero

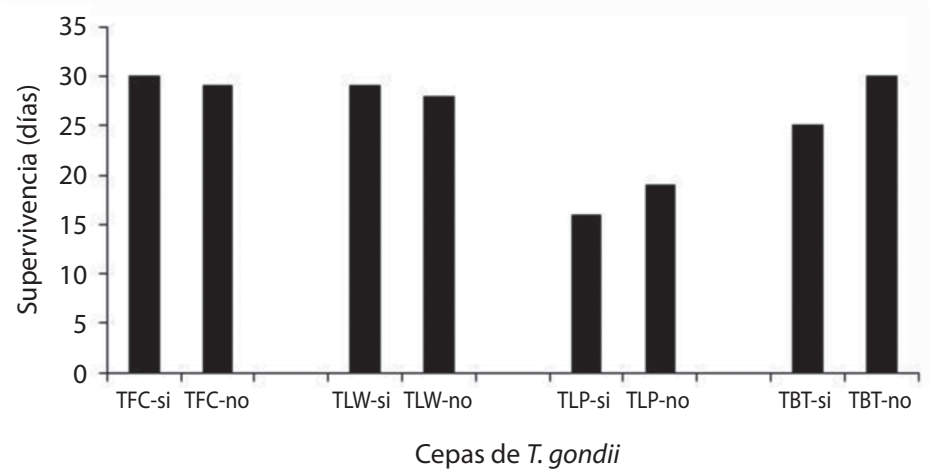

Fig. 3. Supervivencia de ratones infectados con diferentes cepas de T. gondii previa inoculación con T. musculi. Fig. 3. Survival time of mice infected with different strains of $T$. gondii after T. musculi inoculation.

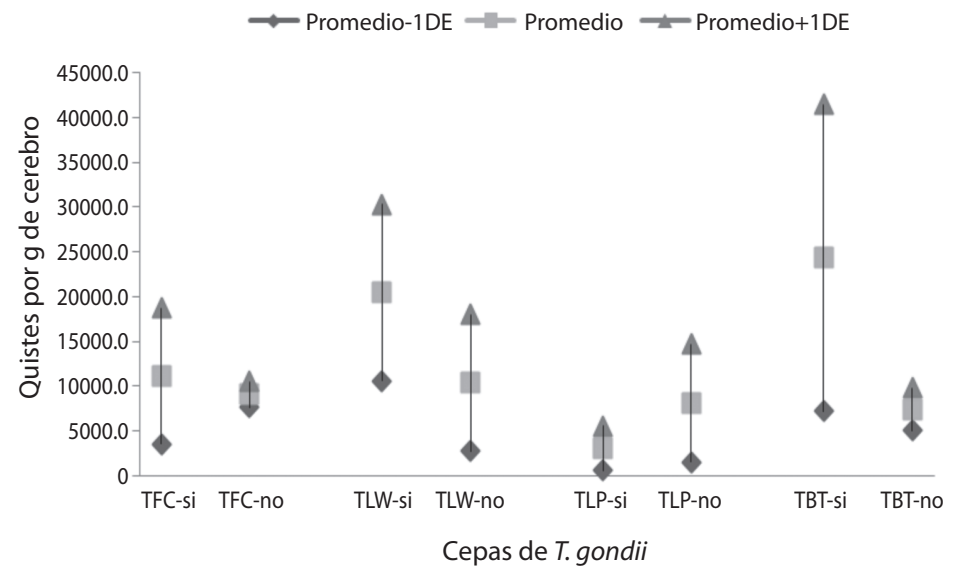

Fig. 4. Número de quistes en el cerebro de ratones infectados con diferentes cepas de T. gondii con o sin previa infección con T. musculi.

Fig. 4. Number of T. gondii cysts found in the brain of mice infected with different strains after T. musculi inoculation. 
también por parásitos. Dentro de estos últimos, se han observado estos procesos en infecciones de organismos de mayor tamaño como lo son las especies del género Schistosoma (Capron \& Capron 1986), así como Strongyloides stercoralis (Ruano et al. 2005, Basile et al. 2010, Corti et al. 2011) entre otros. Sin embargo, el mayor impacto se observa en parásitos que por su naturaleza intracelular, o su acción directa interna sobre órganos vitales, son profundamente afectados por cualquier alteración en la inmunidad celular, blanco fundamental de la inmunosupresión (Ferreira \& Borges 2002, Dedet \& Pratlong 2005). Tal es el caso de los tripanosomas del grupo Salivaria (africanos) (Uzonna et al. 1998), (Trypanosoma cruzi (Krettli 1977, Sztein \& Kierszenbaum 1993, Bacal et al. 2010), Leishmania spp. (Oliveira et al. 2008), Toxoplasma gondii (Abedalthagafi et al. 2009, García et al. 2010, Soléne et al. 2010) y Plasmodium falciparum (Grimwade et al. 2004). El ejemplo más llamativo causante de este proceso son las infecciones por VIH (Raju et al. 2008, Cruz et al. 2006, Otieno et al. 2006, Jayawardena et al. 2008) en las cuales el curso de la enfermedad es más severo producto del debilitamiento del sistema inmune. En el caso del T. gondii cuya manifestación usual es una infección crónica, la infección concomitante con VIH desencadena una toxoplasmosis diseminada, con graves efectos clínicos sobre algunos pacientes (Davarpanah et al. 2007, Soléne et al. 2010).

Los conocimientos que se puedan aportar al estudio de este fenómeno inmunológico pueden ser de utilidad científica y práctica, en el análisis del proceso y sus consecuencias en el campo médico. En este sentido, el establecimiento de modelos experimentales, aunque no necesariamente extrapolables al modelo humano, representan una ayuda importante para realizar tal análisis. En los estudios previamente realizados usando T. lewisi como el organismo capaz de exacerbar las infecciones por $T$. gondii (Guerrero et al. 1997, Chinchilla et al. 2004) en las ratas blancas, se observan en los animales sobrevivientes lesiones pulmonares muy similares, desde el punto vista patológico, a las que se producen en los individuos infectados con VIH (Catarinella et al.1998). Datos como estos y otros como la inhibición del interferón gamma durante el proceso (Chinchilla et al. 2005) o el efecto que se manifiesta inclusive a nivel celular en macrófagos peritoneales (Catarinella et al 1999, Chinchilla et al. 2004) y alveolares (Ríos et al. 2009), constituyen información inmunológica básica que eventualmente puede ser importante en la comprensión de estos fenómenos.

Los resultados obtenidos en el modelo expuesto en este estudio, usando también $T$. gondii pero sustituyendo el T. lewisi por el $T$. duttoni, parásito de ratones, reflejan un efecto inmunosupresor similar tanto al analizar la supervivencia como al determinar el número de quistes por gramo de cerebro en los animales sobrevivientes. Al igual que en el modelo con ratas (Guerrero et al. 1997), en este estudio se nota que el efecto inmunosupresor se manifiesta en forma más clara cuando la infección previa con el tripanosomátido se realiza cuatro días antes, disminuyendo el efecto conforme el tiempo previo es más prolongado. Este efecto probablemente es causado por la inhibición de interferón gamma, muy importante en el bloqueo de las infecciones agudas por $T$. gondii (Zhao et al. 2007), así demostrado por el modelo con la rata blanca (Chinchilla et al. 2005).

La cantidad de tripanosomas inoculados no parece ser un factor importante en la mayor o menor manifestación del efecto, tal y como se observa en los datos de supervivencia que se presentan en nuestros resultados. El número de quistes de $T$. gondii inoculados en este caso fue un poco más elevado, con el objetivo de observar mejor las diferencias en supervivencia en relación con el control como realmente ocurrió. Además, la observación de que la diferencia en cuanto al número de quistes en el cerebro entre el grupo control y en los pocos animales que sobrevivieron fue bastante marcada, independientemente del inóculo del tripanosoma, nos indica que el factor que induce el efecto está en el parásito o es liberado por éste, y que presenta una actividad tal, que solo necesita la reproducción del mismo durante cuatro días 
para ejercer su acción inhibidora. Productos derivados de protozoarios con esta capacidad inmunosupresora han sido encontrados en tripanosomas africanos (Darji et al. 1992, 1996), así como para T. lewisi (Ndarathi 1991).

El hecho de que en los experimentos con cepas diferentes del $T$. gondii, se observara la inhibición inmunitaria para cualquier cepa, tanto en la supervivencia de los animales como en el número de quistes en el cerebro de los sobrevivientes, confirma que el factor inmunosupresor no es específico, sino que es un proceso inmune generado por el tripanosomátido contra el $T$. gondii a nivel celular como ha sido demostrado en otros estudios (Mabbott et al. 1995). En el caso de T. lewisi por ejemplo, el efecto fue demostrado también para infecciones en ratas blancas de macrófagos alveolares por Cryptococcus neoformans (Gross et al. 2006), un organismo muy lejano desde el punto de vista biológico y taxonómico del T. gondii.

Este nuevo modelo, aunque similar al de T. lewisi ya publicado, tiene la ventaja de que emplea un animal de más fácil manejo, el ratón blanco (Mus musculus), en comparación con la rata blanca (Rattus rattus). Este aspecto junto con el aporte para una mejor comprensión de algunos procesos inmunológicos, propios de las enfermedades debilitantes del ser humano, es el aporte de este trabajo. Sin embargo, quedan varias dudas en el sentido de determinar si este efecto se puede manifestar también a nivel celular, como se ha demostrado específicamente en macrófagos peritoneales (Catarinella et al. 1999, Chinchilla et al. 2004) o alveolares (Ríos et al. 2009), o el papel de las linfoquinas en el proceso. Estudios en ambos sentidos se encuentran en proceso.

En conclusión, este estudio ha aportado los siguientes elementos: 1. La presentación de un modelo de inmunosupresión entre dos parásitos de diferente escala zoológica, uno de ellos $T$. gondii de importancia médica. 2. Datos que podrían contribuir para el mayor conocimiento de los procesos de inmunosupresión producidos por las enfermedades debilitantes y 3 . El uso de un animal de laboratorio, el ratón, de más fácil manejo, en este tipo de estudios inmunológicos.

\section{AGRADECIMIENTOS}

Este estudio fue realizado con el apoyo del departamento de Investigación de la Universidad de Ciencias Médicas (UCIMED), del Ministerio de Ciencia y Tecnología (MICIT) y del Consejo Nacional para Investigaciones Científicas y Tecnológicas (CONICIT). Agradecemos a Laura Valerio, José Bolaños y Edwin Valenciano por el manejo y mantenimiento de los animales de laboratorio y a Juan Carlos Vanegas por el apoyo brindado en los análisis estadísticos y confección de figuras.

\section{RESUMEN}

La prevalencia de infecciones por Toxoplasma gondii en el ser humano es de 5-90\% según la zona geográfica; en Costa Rica por ejemplo, la seroprevalencia es de un 58\%, por lo que es importante comprender algunos procesos inmunológicos, propios en estas afectaciones parasitarias. Con el objeto de determinar si el Trypanosoma musculi ejerce procesos de inmunosupresión sobre Toxoplasma gondii se realizó un experimento en el que se inocularon ratones Swiss con T. musculi cuatro, cinco, seis y siete días previos a la infección con $T$. gondii, ocurriendo la inmunosupresión cuando la inoculación con T. musculi fue hecha cuatro días antes. Además, la cantidad de tripomastigotos inoculados no influyó en el proceso. Se probaron tres cepas de T. gondii aisladas de las heces de un gato casero (TFC), de un Leopardus pardalis (TLP), de un Leopardus wiedii y de la carne de un Bos taurus (TBT). La cepa TLP resultó ser muy patógena, matando a los animales en un tiempo corto, independientemente de la inoculación con $T$. musculi; para las otras cepas se mantuvo el patrón de inmunosupresión en los ratones. Se reporta entonces un modelo experimental de inmunosupresión, aspecto muy en boga en este momento, por su relación con enfermedades que inducen esta condición en el ser humano, especialmente a enfermedades como el cáncer y el SIDA. Este modelo es más fácil de aplicar experimentalmente que el correspondiente con T. lewisi previamente descrito, el cual usa ratas blancas de más difícil manejo que los ratones usados en este estudio.

Palabras clave: Toxoplasma gondii, inmunosupresión, Trypanosoma musculi, modelo experimental, inmunidad. 


\section{REFERENCIAS}

Abedalthagafi, M.E., J. Rushing, D. Garvin, B. Cheson \& M. Ozdemirli. 2009. Asymptomatic diffuse "encephalitic" cerebral toxoplasmosis in a patient with chronic lymphocytic leukemia: case report and review of the literature. Int. J. Clin. Exp. Pathol. 3: 106-109.

Albright, J.W. \& J.F. Albright. 1991. Rodent trypanosomes: their conflict with the immune system of the host. Parasitol. Today 7: 137-140.

Bacal, F., C.P. Silva, P.V. Pires, S. Mangini, A.I. Fiorelli, N.G. Stolf \& E.A. Bocchi. 2010. Transplantation for Chagas disease: an overview of immunosuppression and reactivation in the last two decades. Clin. Transplant. 24: E29-E34.

Basile, A., S. Simzar, J. Bentow, F. Antelo, P. Shitabata, S.K. Peng \& N. Craft. 2010. Disseminated Strongyloides stercoralis: hyperinfection during medical immunosuppression. J. Am. Acad. Dermatol. 63: 896-902.

Bojar, I. \& J. Szymanska. 2010. Environmental exposure of pregnant women to infection with Toxoplasma gondii- state of the art. Ann. Agric. Environ. Med. 17:209-214.

Capron, M. \& A. Capron. 1986. Rats, mice and men models for immune effectors mechanisms against schistosomiasis. Parasitol. Today 2: 69-75.

Catarinella, G., M. Chinchilla, O.M. Guerrero \& E. Abrahams. 1998. Effect of Trypanosoma lewisi (Kinetoplastida; Trypanosomatidae) on the infection of white rats with Toxoplasma gondii (Eucoccidia: Sarcocystidae) oocysts. Rev. Biol. Trop. 46: 1121-1123.

Catarinella, G., M. Chinchilla, O.M. Guerrero \& A. Castro. 1999. Infection of white rats peritoneal macrophages wiht Toxoplasma gondii (Coccidia: Sarcocystidae) after Trypanosoma lewisi (Kinetoplastida: Trypanosomatidae) infection. Rev. Biol. Trop. 47: 483-488.

Chinchilla, M., M. Alfaro \& O.M. Guerrero. 1981. Adaptación natural de rata blanca a Toxoplasma gondii. Rev. Biol. Trop. 29: 273-282.

Chinchilla, M., O.M. Guerrero \& A. Castro. 2004. Effect of Trypanosoma lewisi infection on the Toxoplasma gondii multiplication in white rat peritoneal macrophages. Parasitol. Latinoam. 59: 3-7.

Chinchilla, M., O.M. Guerrero \& E. Solano. 1982. Lack of multiplication of Toxoplasma in macrophages of rats in vitro. J. Parasitol. 68: 952-955.

Chinchilla M., O. M. Guerrero \& E. Valenciano. 1985. Efecto de los corticosteroides sobre la adaptación natural de la rata blanca al Toxoplasma. Rev. Cost. Cienc. Méd. 6: 113-118.
Chinchilla, M., L. Reyes, O.M. Guerrero-Bermúdez \& A. Castro. 2005. Role of interferon $g$ on the immunosuppression during Toxoplasma gondii infection by Trypanosoma lewisi. Parasitol. Latinoam. 60: 54-56.

Corti, M., M.F. Villafañe, N. Trione, D. Risso, J.C. Abuín \& O. Palmieri. 2011. Infección por Strongyloides stercoralis: estudio epidemiológico, clínico, diagnóstico y terapéutico en 30 pacientes. Rev. Chil. Inf. 28: 217-222.

Cruz, I., J. Nieto, J. Moreno, C. Cañavate, P. Desjeux \& J. Alvar. 2006. Leishmania/HIV co-infections in the second decade. Ind. J. Med. Res. 123: 357-388.

Da Silva, R.C., A.V. Da Silva \& H. Langoni. 2010. Recrudescence of Toxoplasma gondii infection in chronically infected rats (Rattus norvegicus). Exp. Parasitol. 125: 409-412.

Da Silva, A., G. Vilar, A. Santos, R. Rodrigues, M. Santos \& J. Lannes-Vieira. 2010. Trypanosoma cruzi-Induced Central Nervous System Alterations: From the Entry of Inflammatory Cells to Potential Cognitive and Psychiatric Abnormalities. J. Neuroparasitol. 1: $1-13$.

Darji, A., A. Beschin, M. Sileghem, H. Heremans, L. Brys \& P. de Baetselier. 1996. In vitro stimulation of immunosuppression caused by Trypanosoma brucei: active involvement of gamma interferon and tumor necrosis factor in the pathway of suppression. Inf. Immun. 64: 1937-1943.

Darji, A., R. Lucas, E. Magez, J. Torreele, M. Palacios, E. Sileghem, S. Bajyana, R. Hamers \& P. De Baetselier. 1992. Mechanisms underlying trypanosome elicitedimmunosuppression. Ann. Soc. Belge Méd. Trop. 72: $27-38$.

Davarpanah, M.A., D. Mehrabani, R. Neirami, M. Ghahremanpoori \& M. Darvishi. 2007. Toxoplasmosis in HIV/AIDS patients in Shiraz, southern Iran. Iran. Red Crescent Med. J. 9: 22-27.

Dedet, J.P. \& F. Pratlong. 2005. Leishmania, Trypanoso$m a$ and Monoxenous Trypanosomatids as Emerging Opportunistic Agents. J. Euk. Microbiol. 47: 37-39.

Dubey, J.P. \& J.L. Jones. 2008. Toxoplasma gondii infection in humans and animals in the United States. Int. J. Parasitol. 38: 1257-1278.

Duncan, M.D. \& D.S. Wilkes. 2005. Transplant-related Immunosuppression. A Review of Immunosuppression and Pulmonary Infections. Proc. Am. Thor. Soc. 2: 449-455.

Elmore, S.A., J.L. Jones, P.A. Conrad, S. Patton, D.S. Lindsay \& J.P. Dubey. 2010. Toxoplasma gondii: epidemiology, feline clinical aspects and prevention. Trends Parasitol. 26: 190-196. 
Fazzani, C., P.A. Guedes, A. Senna, E.B. Souza, H. Goto \& J.A.L. Lindoso. 2011. Dynamics of immunosuppression in hamsters with experimental visceral leishmaniasis. Braz. J. Med. Biol. 44: 666-670.

Ferreira, M.S. \& A.S. Borges. 2002. Some Aspects of Protozoan Infections in Immunocompromised Patients - A Review. Mem. Inst. Oswaldo Cruz 97: 443-457.

Garcia de la Fuente, I., M. Ansari, A.L. Rougemont, R.J. Passweg, F. Gumy-Pause, H. Ozsahin, J. Lobrinus \& K. Posfay-Barbe. 2010. Acute Disseminated Fatal Toxoplasmosis After Haploidentical Stem Cell Transplantation Despite Atovaquone Prophylaxis in A Young Man. Ped. Inf. Dis. J. 29: 1059-1060.

Guerrero-Bermudez, O.M., M. Chinchilla-Carmona \& A. Castro-Castillo. 1995. Age influence in the natural resistance of white rat and mice to the protozoan Toxoplasma gondii. Rev. Biol. Trop. 43: 1-3.

Guerrero, O.M., M. Chinchilla \& E. Abrahams. 1997. Increasing of Toxoplasma gondii (Coccidia, Sarcocystidae) infections by Trypanosoma lewisi (Kinetoplastida, Trypanosomatidae) in white rats. Rev. Biol. Trop. 45: 877-882.

Grimwade, K., N. French, D.D. Mbatha, D.D. Zungu, M. Dedicoat \& C.F. Gilks. 2004. HIV infection as a cofactor for severe falciparum malaria in adults living in a region of unstable malaria transmission in South Africa. AIDS 2018: 547-554.

Gross, N., O.M. Guerrero, M. Chinchilla \& C. JarstrandHall. 2006. Trypanosoma lewisi-induced immunosupression: The effects on alveolar macrophages activities against Cryptococcus neoformans. Exp. Parasitol. 113: 262-266.

Holland, G.N. 2003. Ocular toxoplasmosis: a global reassessment. Part I: epidemiology and course of disease. Am. J. Ophthalmol. 136: 973-88.

Holst, I. \& M. Chinchilla. 1990. Development and distribution of cysts of an a virulent strain of Toxoplasma and the humoral immune response in mice. Rev. Biol. Trop. 38: 189-93.

Jayawardena, S., S. Singh, O. Burzyantseva \& H. Clarke. 2008. Cerebral Toxoplasmosis in Adult Patients with HIV Infection, p. 17-24. In M.A. Perazella (ed.). Resident Grand Rounds. Series.

Kaňková, Š., V. Holáň, A. Zajícová, P. Kodym \& J. Fleg. 2010. Modulation of immunity in mice with latent toxoplasmosis -the experimental support for the immunosuppression hypothesis of Toxoplasmainduced changes in reproduction of mice and humans. Parasitol. Res. 107: 1421-142.
Krettli, A.U. 1977. Exacerbation of experimental Trypanosoma cruzi infection in mice by concomitant malaria. J. Protozool. 24: 514-518.

Mabbott, N.A., I.A. Sutherland \& J.M. Sternberg. 1995. Suppressor macrophages in Trypanosoma brucei infection: nitric oxide is related to both suppressive activity and lifespan in vivo. Parasite Immunol. 17: 143-50.

Manzano-Alonso, M.L. \& G. Castellano-Tortajada. 2011. Reactivation of hepatitis B virus infection after cytotoxic chemotherapy or immunosuppressive therapy. World J. Gastroenterology 17: 1531-1537.

Ndarathi, C.M. 1991. Suppressor and protector factors derived from Trypanosom lewisi. Int. J. Parasitol. 21: 736-69.

Oliveira, R.A., L.S.V. Silva, V.P. Carvalho, A.F. Coutinho, F.G. Pinheiro, C.G. Lima, J.E. Leandro Jr, G.B. Silva Jr. \& E.F. Daher. 2008. Visceral leishmaniasis after renal transplantation: report of 4 cases in northeastern Brazil. Transplant. Inf. Dis. 10: 364-368.

Otieno, R.O., C. Ouma, J.M. Ongecha, C. Keller, T. Were, E.N. Waindi, M. Michaels, R.D. Day, J.M. Vulule, D.J. Perkins \& J. Douglas. 2006. Increased severe anemia in HIV-1-exposed and HIV-1-positive infants and children during acute malaria. AIDS 20: 275-280.

Raju, R.G., F.E. Chaudhary, F.E. Bilimoria \& S.K. Katare. 2008. Diffuse cutaneous leishmaniasis: Co-infection with human immunodeficiency virus (HIV) Ind. J. Dermatol. Venereol. Leprol. 74: 641-643.

Ríos-Carrera, N.J., M. Chinchilla, O.M. Guerrero \& A. Castro. 2009. Efecto inmunosupresor de Trypanosoma lewisi (Kinetoplastidae) sobre la multiplicación de Toxoplasma gondii (Sarcocystidae) en macrófagos alveolares y peritoneales de rata blanca Rev. Biol. Trop. 57: 13-22.

Ruano, A.L., T. Martin, J. Pardo, J. López-Albán \& A. Muro. 2005. Avances en el estudio de la estrongiloidosis. Enf. Emerg. 7: 102-109.

Sitoe, S.P.B.L., B. Rafael, L.R. Meireles, H.F. de Andrade Jr. \& R. Thompson. 2010. Preliminary report of HIV and Toxoplasma gondii occurrence in pregnant women from Mozambique. Rev. Inst. Med. Trop. Sao Paulo 52: 291-295.

Soléne, P.D., JP. Gangneux, S. Lavoué, B. Lelong, C. Guiguen, Y. le Tulzo \& F.R. Gangneux. 2010. Correlation of Parasite Load Determined by Quantitative PCR to Clinical Outcome in a Heart Transplant Patient with Disseminated Toxoplasmosis. J. Clin. Microbiol. 48: 2541-2545. 
Sztein, M.B. \& F. Kierszenbaum. 1993. Mechanisms of development of immunosuppression during Trypanosoma infections. Parasitol. Today 9: 424-428.

Uzonna, J.E., S.R. Kaushik, Y. Zhang, J.R. Gordon \& H. Tabel. 1998. Experimental murine Trypanosoma congolense infections. II. Role of splenic adherent $\mathrm{CD}^{+}$Thy $1.2^{+}$TCR- $\alpha 3^{-} \gamma \delta^{-} \mathrm{CD} 4^{+} 8^{-}$and $\mathrm{CD}^{+}$ Thy $1.2^{+} \mathrm{TCR}-\alpha \beta^{-} \gamma \delta^{-} \mathrm{CD} 4^{-} 8^{-\mathrm{c}}$ ells in the production of IL-4, IL-10, and IFN- $\gamma$ and in Trypanosome-elicited Immunosuppression. J. Immunol. 161: 6189-6197.

Viens, P.G., A.T. Targett, E. Leuchar \& A.J.S. Davies. 1974. The immunological response of CBA mice to
Trypanosoma musculi. Initial control of the infection and the effect of t-cell deprivation. Clin. Exp. Immunol. 16: 279-294.

Zapata, M., L. Reyes \& I. Holst. 2005. Disminución en la prevalencia de anticuerpos contra Toxoplasma gondii en adultos del valle central de Costa Rica. Parasitol. Latinoam. 60: 32-37.

Zhao, Y., D. Wilson, S. Matthews \& G.S. Yap. 2007. Rapid Elimination of Toxoplasma gondii by Gamma Interferon-Primed Mouse Macrophages is Independent of CD40 Signaling. Infect. Immun. 75: 4799-4803. 